\title{
Concerns regarding sacral neuromodulation as a treatment option for medical-refractory overactive bladder
}

\author{
Daniel Liberman, MD; Luc Valiquette, MD FRCSC \\ University of Montreal Health Centre, Department of Urology, Montreal, QC
}

$\mathrm{S}$ acral neuromodulation (SNM) with Interstim therapy (Medtronic, Minneapolis, MN) has become one of the accepted treatment options for patients with medication-refractory overactive bladder $(\mathrm{OAB})$ with or without incontinence. ${ }^{1}$ While this modality has shown some interesting results, many unanswered questions remain. With the current data available, we do not recommend SNM for OAB patients for the following reasons.

\section{Heterogeneous patient population and vague indications for SNM}

Implanted SNM has been proposed as a treatment option for patients suffering from severe urgency-frequency and urinary urge incontinence (UUI). Three large, randomized controlled trials (RCTs) have corroborated its use. ${ }^{2-4}$ However, Brazelli and colleagues explained that there was a discrepancy in the clinical characteristics of the patients' treatment history prior to the implantation. ${ }^{5}$ Most patients received pharmacological and/or surgical operations, while other patients received only conservative non-surgical therapy. Moreover, there were inconsistencies with the severity and duration of symptoms. Evidence based on heterogeneous patient populations and discrepancies in clinical predictors of success makes it difficult to conclude which patients would truly benefit from this procedure.

\section{Patient selection and surgeon/hospital variability}

The indications for SNM are not absolute and the rate at which the procedure is performed depends on the preference of the surgeon and the wishes of the patient. Significant variability exists with the use of this treatment modality. By reviewing administrative billing data, Cameron and col- leagues reported the success rates of SNM in the United States, including Medicare beneficiaries $(n=1490)$ and privately insured individuals $(n=1060) .{ }^{6}$ The authors concluded that success rates in the community were significantly lower to those in published case series and RCTs. The overall mean success rate was $39.9 \%$ and $49.1 \%$ in the Medicare sample and the privately insured, respectively. This was significantly lower than the $88 \%$ reported and the $52 \%$ to $77 \%$ rates of success for the Brazzelli and Siddiqui studies, respectively. ${ }^{5,7}$ Selection criteria and experience of the surgeon clearly affects success. Furthermore, RCTs and single institution case series have been limited to experts with better patient selection and availability of state of the art technology. Van Voskuilen and colleagues reported a surgical re-intervention rate of $48 \%$ in 149 patients with SNM at a mean follow-up of 64.2 months and correlated the high reoperation rate to a considerable learning curve in patient selection and surgical technique. ${ }^{8}$ Most studies did not include information on the level of expertise of the clinicians performing the procedure. Moreover, the backup facilities of the hospital or the clinic where the procedures were performed were never described. Therefore, SNM cannot be recommended to large heterogeneous populations and should only be considered after counselling patients realistically about their chances of success and potential complications.

\section{Criteria for success and lack of long-term follow-up}

Clinical success has been defined as an improvement of $50 \%$ or more in symptoms during testing. This definition seems somewhat arbitrary and problematic. Some patients may just attain the threshold of being classified as responders to SNM (i.e., $>50 \%$ response), while other patients may have a substantially greater response. Bosch addressed this limitation. He stated that a report of success using a binary endpoint for testing and after implantation based on a more 
Liberman and Valiquette

than $50 \%$ threshold grossly overestimates the benefits of this treatment. ${ }^{9}$ The clinical heterogeneity in endpoints have rarely been discussed or addressed. A 50\% improvement, while receiving pharmacological therapy, would hardly be considered a success by most patients, especially if they are suffering from $\mathrm{OAB}$ or $\mathrm{UUI} .{ }^{10}$ Results using a more stringent definition for SNM success would be valuable.

An important consideration was that not all patients who were satisfied with the SNM testing underwent implantable pulse generator (IPG) insertion. About $7 \%$ to $10 \%$ of patients, who expressed improvement in symptoms when tested, did not undergo IPG implantation. ${ }^{11,12}$ Moreover, $20 \%$ of successful peripheral nerve evaluation (PNE) will not experience the same efficacy after their permanent lead placement supporting the use of a tined lead. ${ }^{9}$

Few studies had long-term follow-up data in outcome and complications. This is an important consideration given that follow-up has been found to be the best predictor of revision and explantation. ${ }^{13}$ In case series with follow-up longer than 3 years, total failure rates ranged from $29 \%$ to $40 \%{ }^{5}$ In a 14 year experience from a Canadian centre, the explantation rate was $20.8 \%$, and $39 \%$ of patients in this study needed revision of their SNM implant, ${ }^{14}$ comparable to other worldwide clinical studies. ${ }^{12}$ Loss of efficacy of the SNM implant was the most common reason for explantation. It was interesting to note that revision occurred late after implantation, with a median time to revision of 26.5 months. ${ }^{14}$ This evidence strengthens the argument that longterm data must be generated to justify SNM use in the community outside the context of clinical trials.

\section{Adverse events}

A discussion of the therapeutic advantages of SNM cannot be addressed without first interpreting its potential complications. Pain at the stimulation site $(15.3 \%)$, new pain $(9 \%)$, pain at the lead site $(5.4 \%)$, suspected lead migration $(8.4 \%)$, infection $(6 \%)$, transient electric shock $(5.5 \%)$ and changes in bowel function (3\%) have all been reported complications of a permanent implantation after a PNE testing. ${ }^{3}$ Leong and colleagues, in a single centre sample survey, reported that $56 \%$ of patients reported having discomfort at the site of implantation, while a significant proportion of patients experienced problems with metal detectors. ${ }^{15}$ These complication rates must be reported to patients during informed consent.

\section{Cost-benefit analysis}

Sacral neuromodulation is costly and this must be considered. Implanted hardware alone costs $€ 8400$ per implant. Significant additional costs of pre-implantation procedures and surgical revision have also been reported. ${ }^{16}$ The pulse generator has an expected battery life of up to 7 years at which point it will need replacement at a cost of $€ 5300$. Bosch and colleagues conducted a prospective study in patients with refractory UUI and included a health technology assessment that showed that a break-even point for cumulative cost of SNM per patient versus the costs of continued conservative management was only reached at 18.2 years. ${ }^{17}$ Since the cure rates are mostly unknown, the true cost-benefit ratio of this expensive type of treatment remains unknown. ${ }^{10}$

\section{Conclusion}

Although the precise mode of action remains unknown, SNM has been recommended for patients, suffering from urgency-frequency symptoms and UUI, who have failed conservative measures. Although results have shown its potential benefits, uncertainty exists as to the best techniques and the best stimulation settings. Furthermore, the biological effects have been demonstrated in very heterogeneous patient groups with soft definitions of success and unclear cure rates.

For patients with $\mathrm{OAB}$ with or without incontinence who have failed anticholinergic therapy, botulinum toxin A (BTX A) injections has recently been shown to offer an effective alternative to neuromodulation in a double-blind placebo controlled trial..$^{18}$ Therefore the risks and benefits of SNM could be considered as being a last resort before surgical urinary diversion, invasive catherization or long-term use of containment devices, such as incontinence pads. However, the authors believe that the indications for the use SNM will become more restricted and its use will be replaced by more minimally invasive and cost-effective modalities including intravesical BTX A.

Competing interests: None declared.

This paper has been peer-reviewed.

\section{References}

1. Abrams P, Andersson KE, Birder L, et al. Fourth International Consultation on Incontinence Recommendations of the International Scientific Committee: Evaluation and treatment of urinary incontinence, pelvic organ prolapse, and fecal incontinence. Neurourol Urodyn 2010;29:213-40.

2. Schmidt RA, Jonas $U$, Oleson $K A$, et al. Sacral nerve stimulation for treatment of refractory urinary urge incontinence. Sacral Nerve Stimulation Study Group. J Urol 1999;162:352-7.

3. Siegel SW, Catanzaro F, Dijkema, H. E. et al. Long-term results of a multicenter study on sacral nerve stimulation for treatment of urinary urge incontinence, urgency-frequency, and retention. Urology 2000;56:87-91.

4. Hassouna MM, Siegel SW, Nyeholt AA, et al. Sacral neuromodulation in the treatment of urgency-frequency symptoms: a multicenter study on efficacy and safety. J Urol 2000;163:1849-54.

5. Brazzelli M, Murray A, Fraser C. Efficacy and safety of sacral nerve stimulation for urinary urge incontinence: a systematic review. J Urol 2006; 175:835-41.

6. Cameron AP, Anger JT, Madison R, et al. National trends in the usage and success of sacral nerve test stimulation. J Urol 2011;185:970-5. Epub 2011 Jan 19. 
7. Siddiqui NY, Wu JM, Amundsen CL. Efficacy and adverse events of sacral nerve stimulation for overactive bladder: A systematic review. Neurourol Urodyn 2010;29:S18-23.

8. van Voskuilen AC, Oerlemans DJ, Weil EH, et al. Long term results of neuromodulation by sacral nerve stimulation for lower urinary tract symptoms: a retrospective single center study. Eur Urol 2006:49:366-72.

9. Bosch JL. An update on sacral neuromodulation: where do we stand with this in the management of lower urinary tract dysfunction in 2010? BJU Int 2010;106:1432-42.

10. Reynolds WS, Bales GT. Re: Results of sacral neuromodulation therapy for urinary voiding dysfunction: outcomes of a prospective, worldwide clinical study. P. E. van Kerrebroeck, A. C. van Voskuilen, J. P. Heesakkers, A. A. Lycklama a Nihholt, S. Siegel, U. Jonas, C. J. Fowler, M. Fall, J. B. Gajewski, M. M. Hassouna, F. Cappellano, M. M. Ehilali, D. F. Milam, A. K. Das, H. E. Dijkema and U. van den Hombergh. J Urol 2007; 178:2029-34. J Urol 2008; 179:2483-4.

11. Kessler $T M$, Buchser E, Meyer S, et al. Sacral neuromodulation for refractory lower urinary tract dysfunction: results of a nationwide registry in Switzerland. Eur Urol 2007;51:1357-63.

12. van Kerrebroeck PE, van Voskuilen AC, Heesakkers JP, et al. Results of sacral neuromodulation therapy for urinary voiding dysfunction: outcomes of a prospective, worldwide clinical study. J Urol 2007; 178:2029-34.

13. Blandon RE, Gebhart JB, Lightner DJ, et al. Re-operation rates after permanent sacral nerve stimulation for refractory voiding dysfunction in women. BJU Int 2008;101:1119-23.
14. At-Zahrani AA, Elzayat EA, Gajewski JB. Long-term outcome and surgical interventions affer sacral neuromodulation implant for lower urinary tract symptoms: 14-year experience at 1 center. J Urol 2011;185:981-6.

15. Leong RK, Marcelissen TA, Nieman FH, et al. Satisfaction and patient experience with sacral neuromodulation: results of a single center sample survey. J Urol 201 1;185:588-92

16. Bolton JF, Harrison SC. Neuromodulation 10 years on: how widely should we use this technique in bladder dysfunction? Curr Opin Urol 2009: 19:375-9.

17. Bosch JL, Groen J. Sacral nerve neuromodulation in the treatment of patients with refractory motor urge incontinence: long-term results of a prospective longitudinal study. J Urol 2000;163:1219-22.

18. Dmochowski R, Chapple C, Nitit VW, et al. Efficacy and safety of onabotulinumtoxinA for idiopathic overactive bladder: a double-blind, placebo controlled, randomized, dose ranging trial. J Urol 2010;184:2416-22.

Correspondence: Dr. Luc Valiquette, Professor and Director of the Department of Surgery, University of Montreal Hôpital Saint-Luc - Pavillon Principal (porte 4204), Montréal, QC H2X 3J4; fax: 514412-7411; luc.valiquette.chum@ssss.gouv.ca 